Classification

Physics Abstracts

61.16 $\mathrm{Di}-61.505 \mathrm{r}-61.70 \mathrm{r}$

\title{
Theoretical image processing of simulated supported particles (1-3 nm)
}

\author{
Geneviève Nihoul \\ G.M.E.T., Groupe Matériaux, University of Toulon, B.P. 132, 83957 La Grade Cedex, France \\ (Received 13 January 1992, accepted 20 February 1992)
}

\begin{abstract}
Résumé. - Les images de nanoparticules obtenues par HRTEM sont souvent difficiles à interpréter. La petite taille des particules, l'existence de franges de Fresnel et la présence de films stabilisateurs de carbone contribuent à déformer les images. Les résultats quantitatifs sur la taille et la morphologie des particules doivent être dégagés des images dont l'interprétation directe est difficile (et risquée). Le traitement d'images est utilisé pour améliorer la visibilité des particules et de leur interface avec le substrat. Néanmoins, il est bien connu que le traitement d'images peut aussi introduire des nombreux artefacts. Nous présentons ici l'étude de 3 filtres de Fourier différents dont les possibilités et limites ont été testées sur des images simulées de façon à pouvoir les utiliser, sûrement, sur des images expérimentales de nanoparticules.
\end{abstract}

\begin{abstract}
The images of supported nanoparticules obtained by High Resolution Transmission Electron Microscopy are often difficult to interpret. The small size of the particles, the existence of Fresnel fringes and the presence of a stabilizing carbon film all contribute to blur the images. Quantitative results on the size and the morphology of the particles or information about the interface substrate-particle have to be extracted from the images as a direct interpretation is difficult (and hazardous). Simulation of images, starting from a model and computing all the changes in the electron wave introduced by the specimen and the microscope, is a great help to understand the experimental images. Image processing is also used to improve the visibility of the particles and of their interface with their substrate. However, it is well known that image processing can also introduce artefacts in numerous ways. In this paper, we have studied three different Fourier filters: a band pass filter, a Bragg filter and a new filtering by subtraction of the amorphous background. Their possibilities and their limitations, together with their reliability, are tested on computed images in order to use them, safely, on experimental HRTEM images of nanoparticles.
\end{abstract}

\section{Introduction.}

Very small particles, deposited on substrates, are difficult to image by High Resolution Transmission Electron Microscopy (HRTEM) for various reasons. First, their small size (less than $3 \mathrm{~nm}$ ) prevents them from diffracting much and the contrast in the image is rather small. Second, being composed mainly of surfaces, they are surrounded by Fresnel fringes which further blur the image, preventing the surfaces and the interface with the substrate from being clearly viewed; these 
Fresnel fringes depending on the defocus, the apparent size of the particle can vary with the defocus, casting some doubts on a direct measurement of the particle size. Finally, the mobility under the electron beam of the particles on their substrate usually makes it impossible to obtain HREM images. One has to stabilize them by a carbon coating. Though this layer is very thin (about $3 \mathrm{~nm}$ ), it adds a non-negligible background which drowns the already hazy nanoparticles, so that we end up with images where the signal/background ratio is particularly low.

Hence the interpretation of the HREM images has to be done carefully if one wants to get quantitative results from these images. Simulation of images is one method which allows us to study the variation of the image with various parameters. It is not easy to simulate these no-longerperiodic systems, and one has to be very careful in the calculations as spurious effects can easily appear in the simulated image due to a bad choice of computation parameters. Furthermore, we have to include the amorphous carbon contribution in the image if we want to obtain a simulated image which looks like the real one. Image processing is another possibility which can be used along with simulation [1-3]. The simpler possibility is, of course, to increase the contrast by usual methods in the real space. Another interesting possibility is Fourier filtering [4,5]: this can be a great help, not only in getting rid of part of the carbon contribution, but also in erasing most of the Fresnel fringes, as these correspond to low spatial frequencies. One has to use fairly narrow filters as the background is very important and the signal rather low. However, there again, artefacts are easily introduced in a processed image, as the Fourier space is continuous [6]: the continuous part around the main Bragg spots contains all the interesting information about the morphology of the particles and has to be kept, unmodified. It is then necessary to be very careful. A good practice is to use the proposed processing procedure first on simulated images: as we know the model used for the simulation, it is easy to spot the artefacts eventually introduced by image processing.

A certain number of image processing methods have been proposed for small particles: in Fourier space, one uses filters. A band pass ring filter, preserving the interesting spatial frequencies and their surroundings removes part of the amorphous noise and most Fresnel fringe contribution: its width must be carrefully chosen and its edges smoothed. A Bragg filter, keeping only the contribution from around the interesting diffraction spots, seems, at first sight a more interesting system. However, as we will see, in the case of important amorphous background, it introduces ghost lattice images in regions in the image where none are expected. Still in Fourier space, a threshold method has been proposed [7] where all points having an intensity less than a chosen threshold are given a null intensity. This depends strongly on the chosen threshold but gives rise to no ghost fringes. Finally, the single value decomposition method has been applied to small particles [8]. Though it favors one direction of fringes, it can enhance the visibility of the particle morphology.

In this paper, we propose to study the simulation of small particles on a substrate. We will see the influence of the computation parameters and introduce the amorphous contribution. We will also see the variation of the obtained image when several experimental parameters are changed. We will then process some simulated images by various methods; this will allow us to see the possible artefacts inherent to these methods. Finally, we will introduce a new method of Fourier filtering and see its advantages and inconveniences.

\section{Image simulation of small particles.}

The method for computing non-periodic objects such as interfaces, defects, grain boundaries, etc. is well known. One can use the multislice method, dividing the object to be imaged into thin slices which can be considered as bidimensional. However, the use of a computer involves the discretisation of the functions and the introduction of a cut-offf on far values to limit the size 
of the calculations. For periodic objects, the Fourier space is discrete, which gives the sampling in the Fourier space. As the atomic structure factor falls off very quickly with increasing spatial frequency, the cut-off is also easy to find, at least for the potential and transmission functions in the Fourier space. But, for non-periodic objects, one has to introduce an arbitrary period, usually rather large, to prevent edge effects from affecting the interesting object. Hence the defect or small particle in our case is included in a cell large enough for the particle to be far from the edges. This periodisation gives the sampling in the Fourier space, which has to be tight enough to reproduce well the now continuous Fourier space. Finally, the sampling in the real space has to be chosen; as this is linked to the cut-off in the Fourier space, the choice is made according to these two parameters. The size of the matrices used to represent the wave function, the transmission function and the propagation function quickly rises and one has usually to find an acceptable compromise between precision and computer possibilities.

We have computed the images obtained from a model shown in figure $1 \mathrm{k}$ : the particle is a semioctahedron of gold, deposited on a MgO substrate. The lateral size of the particle is $1.6 \mathrm{~nm}$ and its height is $0.8 \mathrm{~nm}$. The gold particle has the $\mathrm{MgO}$ parameters, as has been shown previously [9]. The interface between the particle and the substrate has been supposed perfect, the gold atoms being on the magnesium atoms, as has been shown in the case of nanoparticles of palladium [10]. This particle and its substrate has been included in a square cell $3.36 \mathrm{~nm}$ large. The substrate is, of course, periodic along one of the directions, while it has two surfaces in the other direction. We tried a sampling with $128 \times 128$ points, but this gives a distance between two neighbour points of $0.026 \mathrm{~nm}$ which was thought to be too large. Also, the resulting cut-off in the reciprocal space is too low, as only spatial frequencies less than $18.9 \mathrm{~nm}^{-1}$ were used to reconstruct the potential, and this was obviously not enough. Hence, all the simulations presented here have been done with a $256 \times$ 256 mesh, giving a sampling of $0.013 \mathrm{~nm}$. Then the potential is calculated with spatial frequencies up to $37.8 \mathrm{~nm}^{-1}$. A $512 \times 512$ mesh was used once to ensure that no significant change was seen in the images when a better precision was obtained. The parameters of the microscope are those of the JEOL $4000 \mathrm{EX}$ working at $400 \mathrm{kV}$ : spherical aberration parameter $C_{\mathrm{s}}=1.05 \mathrm{~mm}$, beam divergence $1 \mathrm{mrad}$ and defocus Gaussian spread $10 \mathrm{~nm}$. Defocus series were calculated as the experimental defocus is not well known.

Figures 1a to j show some examples of the obtained images; the defocus is varied along with the position of the particle on the substrate. One can see that the apparent size and visibility of the particle vary quickly with these parameters. All else being equal, a change of $10 \mathrm{~nm}$ in defocus divides the apparent lateral size of the particle by almost 2 (see, for example, Figs. 1a and 1b). Also if the particle is sitting at the bottom or the top of the $\mathrm{MgO}$ cube, its visibility is much changed (see Figs. 1a and 1i).

To better reproduce the experimental images, we have introduced an amorphous component to simulate the effect of the carbon films which effect-exists; films exist in the experimental images, the supporting film and the protecting film. The amorphous component was calculated in the Fourier space, by introducing an amplitude with the experimental radial distribution (taking into account only the two first maxima) and a random phase. This is a crude description, but it is sufficient to study the effect of image processing on the amorphous component of an image. Figure 2 shows some images of an amorphous carbon film and of a supported nanoparticle immersed in amorphous material. One can see that the image is further blurred, that the $\mathrm{MgO}$ crystal has now irregular spots, that the Fresnel fringes are less important and that the particle morphology is even more hidden (or far from the model). These are the images we are going to use to study the effects of the image processing. 


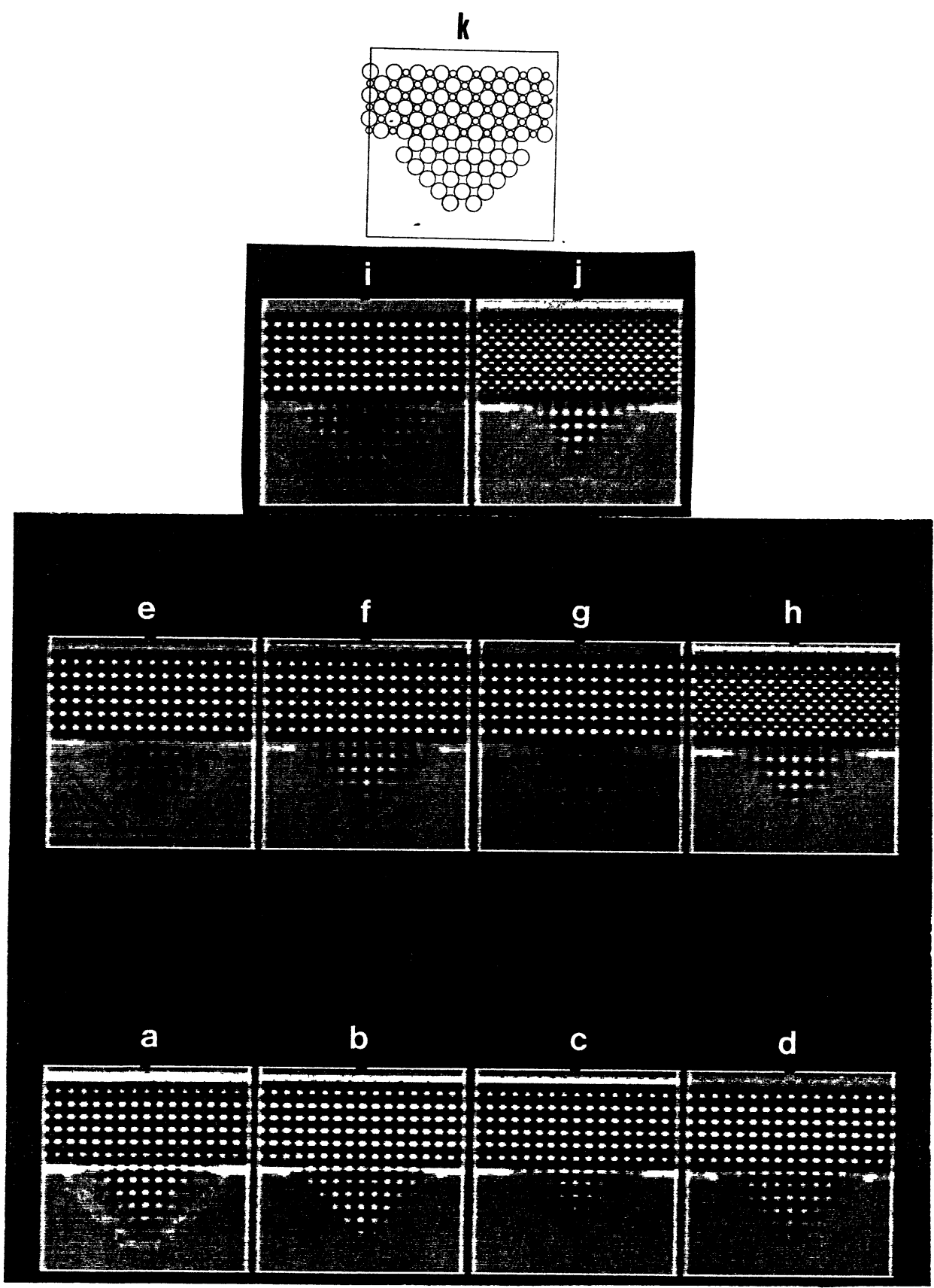

Fig. 1. - This shows some simulated images for different positions of the gold particle along the MgO cube. The notch in the upper part of the frame is a $0.2 \mathrm{~nm}$ scale. For each position, 2 defocusses are shown, $-60 \mathrm{~nm}$ and $-70 \mathrm{~nm}$, measured in reference to the gold cluster. The gold particle is always sitting $2 \mathrm{~nm}$ off the first slice of the $\mathrm{MgO}$ cube. For a) $(-60 \mathrm{~nm})$ and b) $(-70 \mathrm{~nm})$ there is no $\mathrm{MgO}$ after the gold particle. For c) and d) there is $\mathbf{2} \mathrm{nm}$ after it; for e) and f) there is $4 \mathrm{~nm}$ after it; for $\mathrm{g}$ ) and h) there is $6 \mathrm{~nm}$ after it and for i) and j) there is $8 \mathrm{~nm}$ after the gold particle. The appearance of the particle changes very quickly with these two parameters, defocus and particle position. $\mathrm{k}$ ) Model of a half octahedron sitting on a MgO substrate. Both are seen along a [100] direction. The figure is a cut, half way through the half octahedron. The lateral size of the particle is $1.6 \mathrm{~nm}$ and its height is $0.8 \mathrm{~nm}$, while the enclosing cell is $3.36 \mathrm{~nm}$ large. 


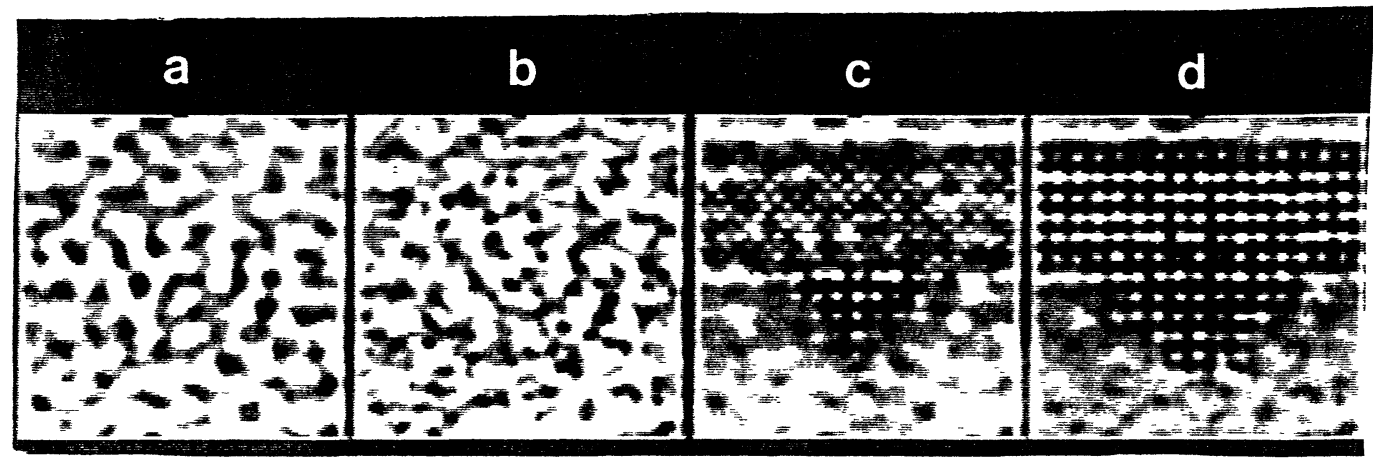

Fig. 2. - a) and b): Computed images of an amorphous carbon film for two defocusses $(-60 \mathrm{~nm}$ and $-70 \mathrm{~nm})$. c) and d): Computed images of a gold particle sitting on a $\mathrm{MgO}$ cube and protected by an amorphous carbon film, for the same defocusses as above.

\section{Image processing.}

The computed images were directly used for different image processing. We will first describe the three processes which have been applied, then show the results.

3.1 FOURIER PROCESSES. - All processes have been applied in Fourier space. Figure 3 shows some Fourier transforms of the images. One can see that there are two different components in the power spectrum: a continuous part, which corresponds to the amorphous part, the Fresnel fringes and all the non-periodic parts of the image; and some spots which correspond to the crystallized part of the image: they are the (200) reflections for $\mathrm{MgO}$ and for gold, plus the spots corresponding to the vector sums of these reflections as the power spectrum is the Fourier transform of the square of the modulus of the wave function. It must be stressed again that all information about the shape and morphology of the particle or the interface is contained in the continuous part of the spectrum surrounding the reflections of the crystallized part. The idea for all image processing is to reduce as much as possible the amorphous and the Fresnel fringe contributions while keeping all information about the crystallized part and its shape, i.e. retain the enlarged Bragg spots, keeping in mind that we don't know their extension and shape.

The first method was to apply the so-called Bragg filters [11]. These take into account not only the interesting spatial frequencies, but also the interesting directions; hence, the filter consists of circular holes, centered on the four (200) reflections. The profiles of the holes have been softened by a Gaussian shape. The filter can then be written as

$$
f(\mathrm{~g})=\exp \left(-\left(\mathrm{g}-\mathrm{g}_{0}\right)^{2} / \sigma^{2}\right)
$$

where $\mathbf{g}$ and $\mathbf{g}_{0}$ are vectors in the Fourier space).

The second method used is to apply a band pass filter centered on the (200) spatial frequency, $g_{0}$ : all spatial frequencies, $g$, near $g_{0}$ are kept; all the others are rejected, whatever their orientation. In order to avoid the artefacts due to sharp edges, a filter with soft edges was used: rather than a Gaussian shape, we have preferred a shape described by equation (2), which gives sharper edges and a flatter maximum (though the results are not very different from the ones obtained with a Gaussian):

$$
f(g)=\exp \left(-\left(g-g_{0}\right)^{4} / \sigma^{4}\right)
$$




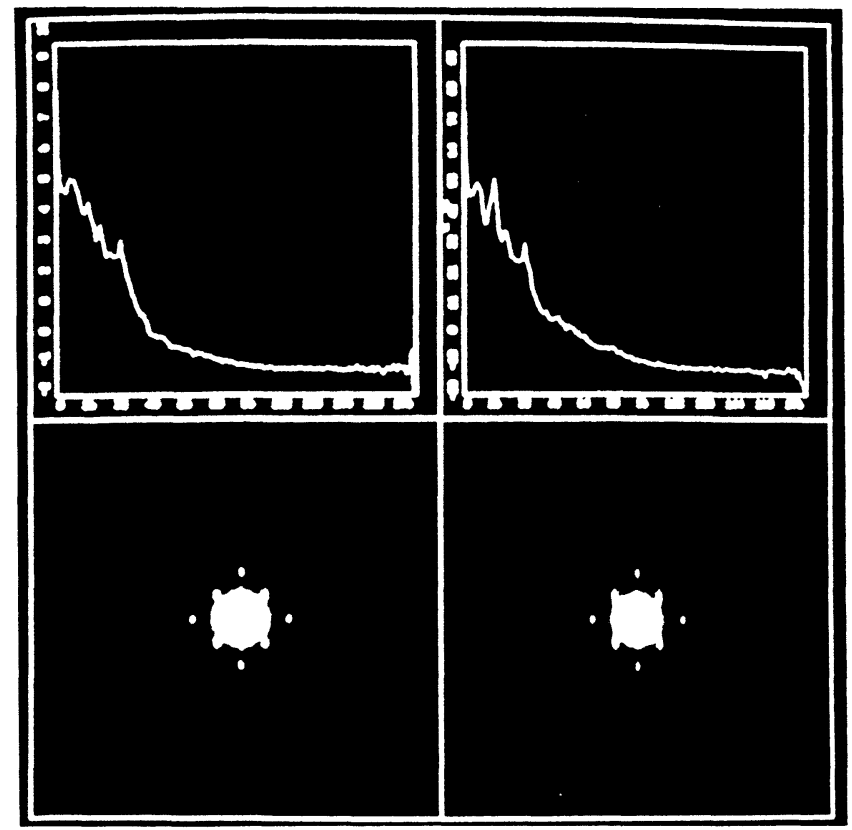

Fig. 3. - Power spectrum of the computed images shown in figures $2 \mathrm{c}$ and $\mathrm{d}$.

where $\sigma$ is a parameter defining the average width of the ring filter, which will be varied.

The third method is a subtraction method. When looking at the radial distribution of the Fourier transform, it is tempting to try to subtract the background due to the amorphous part from the whole signal in order to retain only the crystalline contribution. One can calculate this background for the spatial frequencies around the (200) reflections by averaging the values for points with the same spatial frequencies, but far from the (200) enlarged spots. To do that, we apply a ring-shaped mask, with a varying width and with its mean radius equal to $g(200)$. Four circles are centered on the four (200) reflections; an average of the FT intensity is obtained on all points inside the mask but outside the four circles [12]. Several radii have been tried for these circles: if they are too small, they impinge on the diffraction spots and the results are then very sensitive to their size. We have always taken them rather large and the standard circles have a radius equal to the ring width: in this range of sizes, a change in the value of the radius of the circle does not change the results. To further improve this estimate of the amorphous contribution, we divide the mask into three rings. The average is calculated for the interior and the exterior rings, giving two values $I_{1}$ and $I_{2}$ (see Fig. 4). The value for the medium ring, $I_{3}$, containing the (200) reflections, is taken as the average of $I_{1}$ and $I_{2}$.

This background value being thus estimated, all points outside the four circles are given a null intensity; the points inside the four circles have their intensity compared to $I_{2}:$ if their intensity is smaller, it is assumed that they correspond to an amorphous part and their intensity is put to 0 . If not, several procedures were tried and two were kept: either the intensity is kept as it is, giving a new Fourier distribution, $A(u, v)$, or the background intensity is substracted, $B(u, v)$. In both cases, the phase is kept which is the only possibility as we have no means to measure the background phase. We have tried giving a random phase to the substracted term, but this is not 

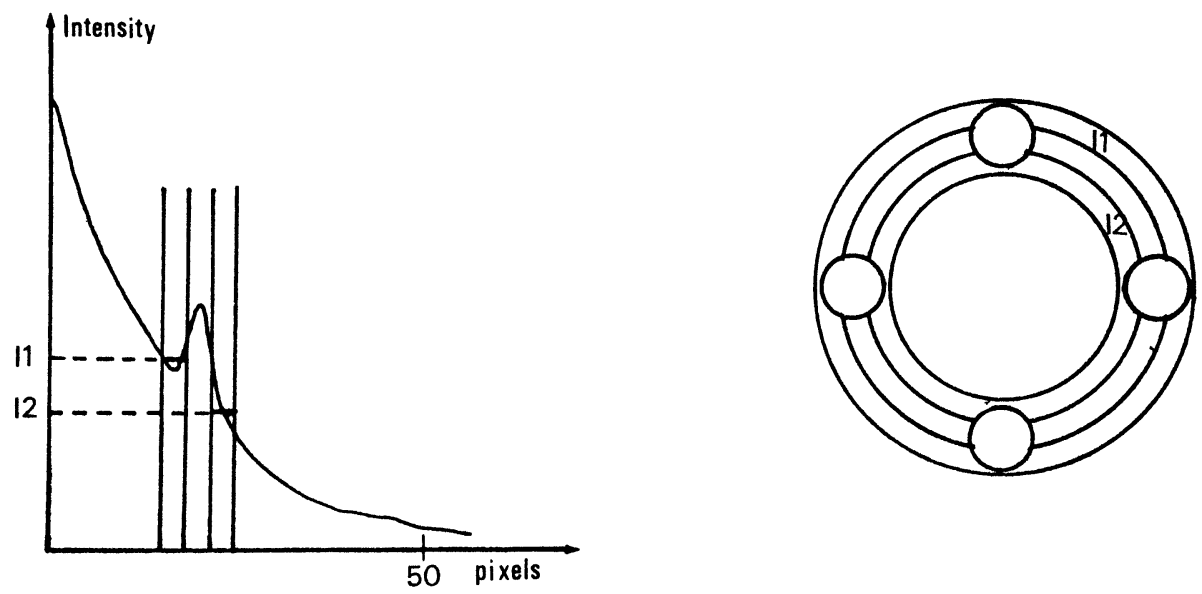

Fig. 4. - Schematic drawing of the radial distribution of a power spectrum, showing the values of $I_{1}$ and $I_{2}$ (see text). b) Rings drawn in the Fourier space, explaining the calculus of the average value of the amorphous contribution for the (200) spatial frequency.

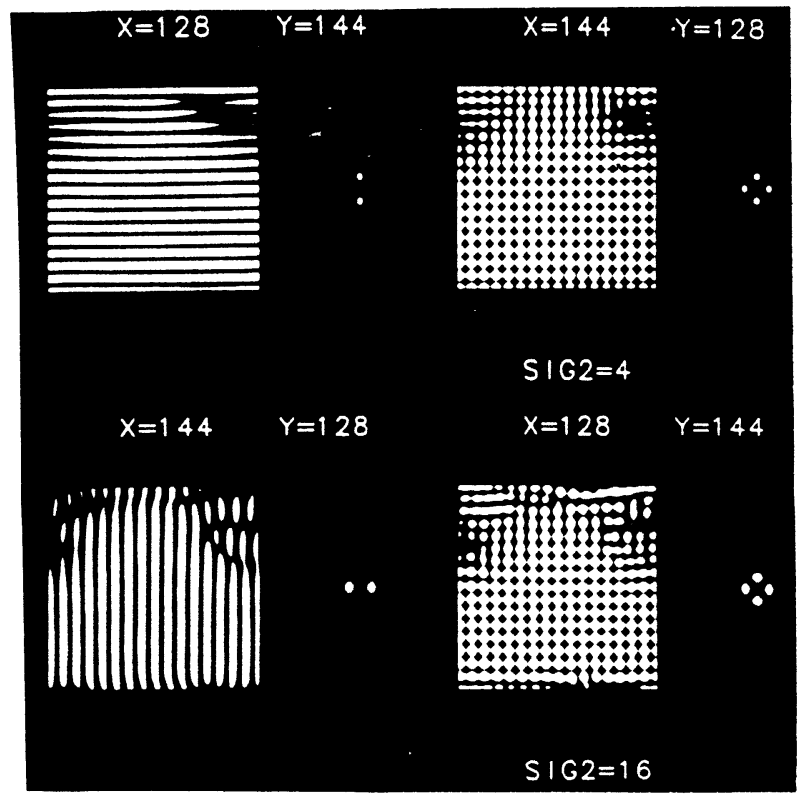

Fig. 5. - Simulated image (2d) processed by Bragg filters, for two different widths. Each time, one can see the results obtained when filtering with only two reflections and then with the four reflections. Ghost lattice images are seen. $\mathrm{X}$ and $\mathrm{Y}$ indicate the coordinates, in pixels, of one of the reflexions. SIG2 is the square of the filter width, $\sigma$.

theoretically more acceptable and, of course, gives rather random results. It is obvious that our procedure does not give a correct result if there is interference between the Bragg beams and the 
amorphous background as can be expected for spatial frequencies close to the Bragg ones. This means that, for this small range of spatial frequencies, we do not substract the background. But for most of the spatial frequencies, our procedure will be correct and, hence, give a non biased substraction of the amorphous contribution.

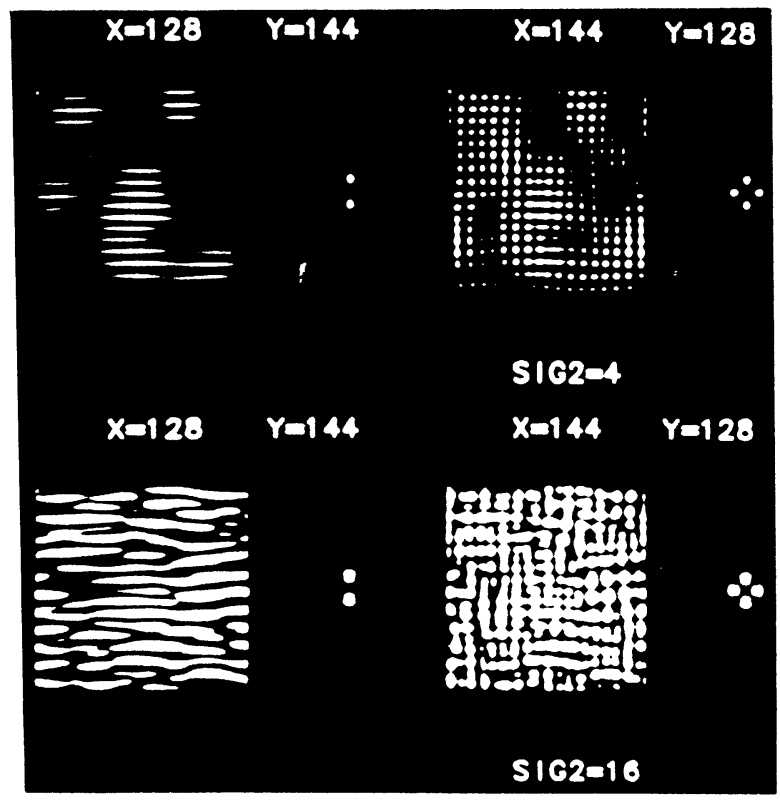

Fig. 6. - Same Bragg filters as above applied on a purely amorphous computed image (Fig. 2b). One sees a crystallized pattern appear with even some dislocations. $X$ and $Y$ indicate the coordinates, in pixels, of one of the reflexions. SIG2 is the square of the filter width, $\sigma$.

\subsection{RESULTS. - We have tried the above described processes on simulated images.}

First, Bragg filters centered on the (200) reflections were used. Figures 5 show some examples for different widths of the filters: one can see that, whatever the width, we have lost the amorphous part but we have gained fringes in parts of the image where there are none! The shape of the gold particle is then more difficult to see than before the processing. This is not surprising as we have forced these periodicities in the Fourier space. To better see this effect, we have processed the purely amorphous images, shown in figures $2 a$ and $b$; one result is shown in figure 6 where the processed amorphous images are shown for two different Bragg filter widths. They look very much like crystallized material with some defects (note, in particular, a dislocation). Hence, we have made some totally non-existent features appear in our image. It explains the previous results on a mixture of crystallized and amorphous material and shows the existence of artefacts introduced by this method.

The band pass filter was then applied to the two simulated images shown in figures $2 \mathrm{c}$ and $\mathrm{d}$. Figures $7 \mathrm{a}$ and $\mathrm{b}$ show the results for several widths. For the smaller image, the width plays an important role as the particle shape changes as the width increases. Obviously, for a small value 

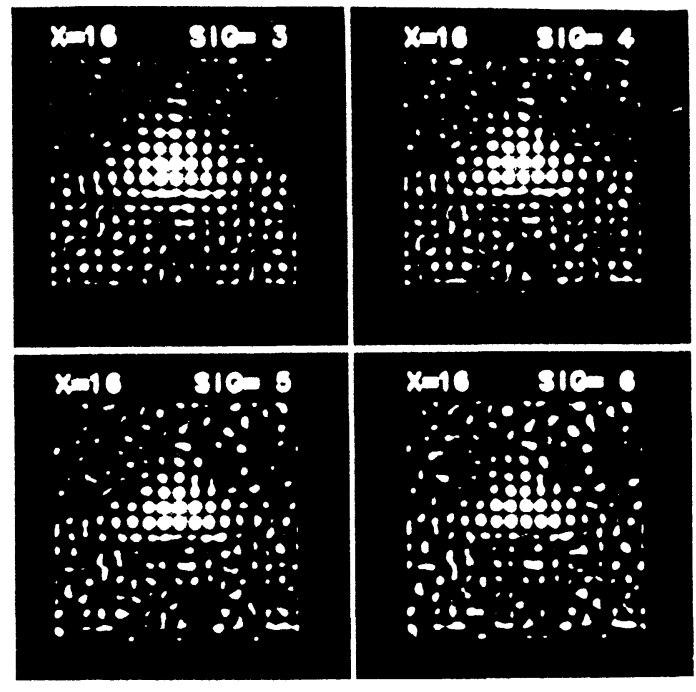

a

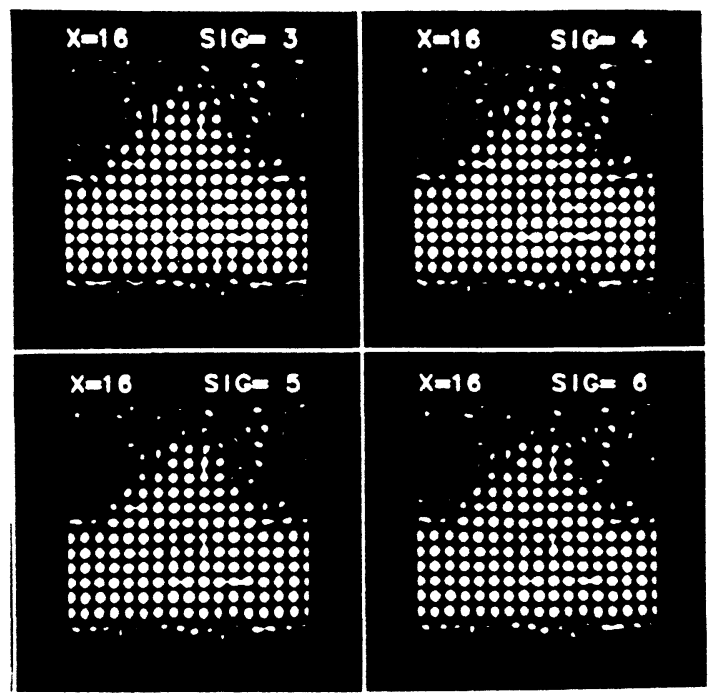

b

Fig. 7. - Simulated images processed with some band pass filters varying in width. a) corresponds to figure $2 \mathrm{c}$ while b) corresponds to figure $2 \mathrm{~d}$. $X$ indicates the average radius of the ring, while SIG is the width of the ring.

of $\sigma$, we cut into the enlarged reflection and lose some information about its shape. For the larger image, this is no longer true, the enlargement being smaller. The shape of the processed particle changes very little as the width finally is doubled. Another interesting effect arises in the smallest image. As the double frequencies were clearly seen in the image $2 c$, which means that for these defocus and thickness the $\mathrm{MgO}$ (200) reflections had a small amplitude, the process, keeping only the (200) frequency, cancels the MgO cube. This could be a useful trick to see where the gold particle stops on an experimental image.

Thus, this band pass filter gives interesting results. The Fresnel fringes have disappeared, in some cases the $\mathrm{MgO}$ crystal can be removed also and the particle appears clearer against the amorphous particle. However, for small particles, the shape seems still distorted from the image.

Finally, we have applied the last proposed process to the same two simulated images (Figs. 2c and d). The results are shown in figures $8 \mathrm{a}$ and $\mathrm{b}$ for several ring widths. One can see that, for both sizes of images, the last widths, where the measurements of $I_{1}$ and $I_{2}$ are obtained by using a larger number of values, give a much lowered background, no Fresnel fringes and a clear shape for the image.

\section{Discussion and conclusion.}

We have first made a study of the appearance of the image, for a given model, when several experimental parameters are changed. This shows that the image can appear much smaller than the original object when the wave function phases change. This is not surprising but can be very annoying for quantitative results. The only solution for this problem seems to be focal series and choosing the largest images. 


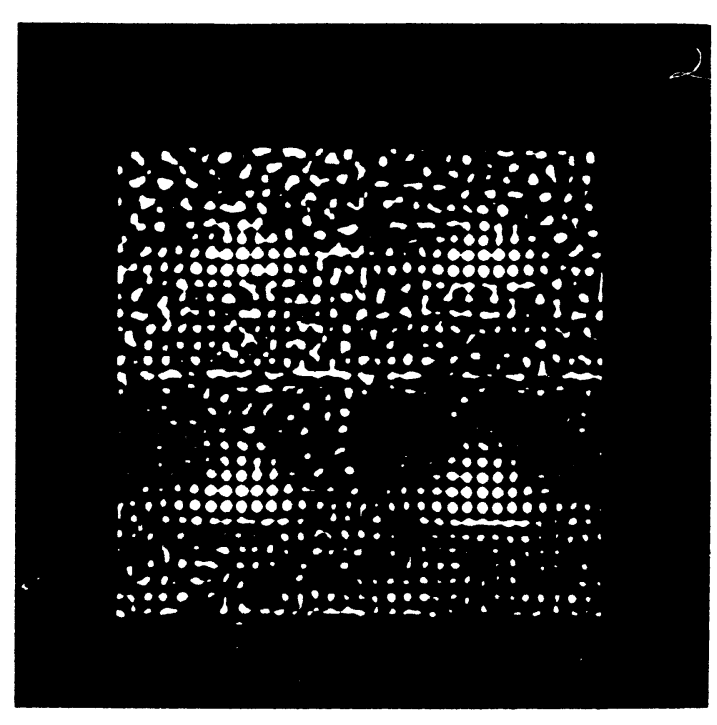

a

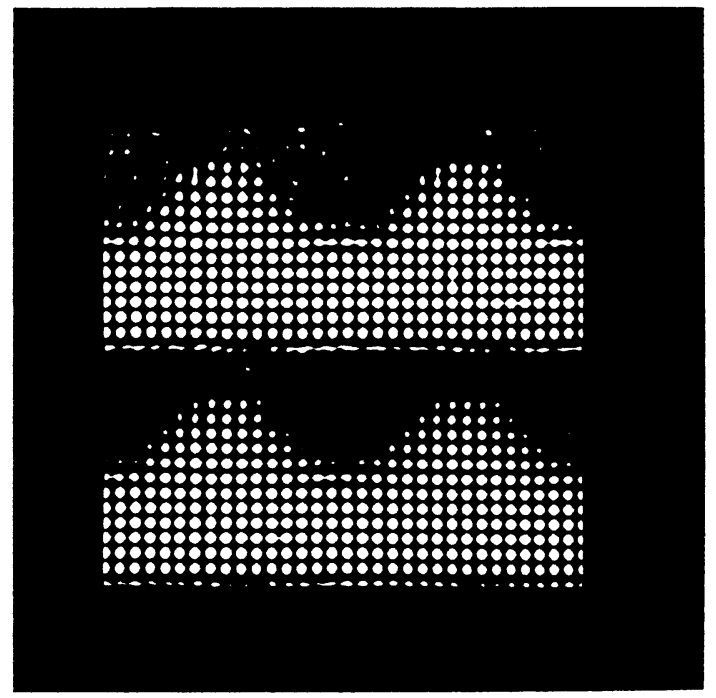

b

Fig. 8. - Simulated images processed with a subtraction of measured background, the widths of the rings used to evaluate the amorphous contribution varying. One can see the particles appear very quickly against a much reduced background. a) corresponds to figure $2 \mathrm{c}$ while $\mathrm{b}$ ) corresponds to figure $2 \mathrm{~d}$.

We have then studied three sorts of image processing which we have tested on computed images. First, we have looked at Bragg filters and we have shown that the existence of amorphous material in our specimens, gave rise to artefacts in the processed images. Hence this kind of filter, though very useful for purely crystallized specimens, can be misleading for mixtures of crystals and amorphous films as it introduces features which were not in the initial image.

The band pass filter avoids this problem of ghost lattices as it does not favor any directions. It is useful to lower the amorphous background and the Fresnel fringes. However, it is very sensitive to the widths of the chosen filter: a too large filter is sure not to impinge on the enlarged Bragg spots but does not subtract much background, while a narrow filter is bound to change the shape of the spot and hence the morphology of the particle or its interface with the substrate.

The last proposed process does seem to keep some of the advantages of both previous methods. It keeps the interesting properties of the Bragg filters but avoids the creation of ghost fringes; it also is more objective as it does not presuppose anything about the shape or size of the enlarged (200) reflection spots, the circles around them being now rather large. Finally, even with these large circles, the subtraction process lowers the background in a very noticeable manner. Therefore we have applied these three methods to experimental particles [13] and are fairly confident in the results obtained by the third method.

Finally, we have shown that, when possible, it can be rewarding to use images where the substrate double frequencies appear, as this can lead, after processing, to a separation of the particle and the substrate contributions to the image. This can be useful in some cases. 


\section{References}

[1] SAXTON W.O., Computed techniques for image processing in electron microscopy (Academic Press; New York, 1978).

[2] SaXTON W.O., Computer processing of electron microscope images, P.W. Hawkes Ed. (SpringerVerlag, 1980, p. 15).

[3] HaWkes P.W., Comp. Graph. Im. Proc. 18 (1982) 58.

[4] FrANCK J., Advanced Technique in Biological Electron Microscopy, J.K. Koehler Ed. (1973) p. 215.

[5] HAWKES P.W., Optik 40 (1974) 539.

[6] SAXTON W.O., Proc. of the 44th meeting of EMSA (1986) p. 526.

[7] PRADÈRE P. and THOMAS E.L., Ultramicroscopy 32 (1990) 149.

[8] ARTal P., AValos BorJa M., SoRia F., POPPa H. and Heinemann K., Ultramicroscopy 30 (1989) 405.

[9] Giorgio S., CHAPON C., HenRY C.R., NiHOUl G. and PÉnisson J.M., Philos. Mag. 64 (1991) 87.

[10] HENRY C.R. and GOYHENE Ch., to be published.

[11] de JONG A.F., COENE W. and VAN DYCK D., Ultramicroscopy 27 (1989) 53.

[12] O'KEEFE M., private communication (1990).

[13] GIORgIO S., NiHOUl G., HENRY C.R. and CHAPON C., Microsc. Microanal. Microstruct. 2 (1991) 627. 